Population Study

Oral

Abstract ID: 140

\title{
Translation, adaptation and psychometric properties of Bahasa Malaysia version of the hearing handicap inventory for adult (HHIA): A preliminary study
}

Tengku Zulaila Hasma Tengku Zam Zam | Ahmad Aidil Arafat Dzulkarnain | Sarah Rahmat | Masnira Jusoh

Kulliyyah of Allied Health Sciences, International Islamic University Malaysia

Introduction: Patient self-perceived handicap inventory is an important tool for modern health care including hearing loss. The HHIA is a self-reporting outcome measurement invented to verify the hearing-impaired patients' complaint and carry out for appropriate client-centered rehabilitation program. To the author's knowledge, none of the self-reported questionnaire for hearing loss is available in Malay language, therefore, further research is required. The aim of this study was to translate the English version of HHIA into Bahasa Malaysia and to psychometrically validate the questionnaire. Methods: The questionnaire was initially translated using forward-backward translation techniques by four-panelists ( 2 panels for each (evel). The translated questionnaire was then reconciled and harmonized for cultural and content by the researchers and two expert panels before being piloted among 10 hearing impaired patients. At the present stage, psychometric evaluations have been conducted among 30 adults hearing-impaired participants using Cronbach's a (for internal reliability) and Pearson's correlation for (inter-item correlation). All participants were recruited from IIUM Hearing and Speech Clinic. Results: Cronbach's a value was 0.96 for combined items. There were a strong correlation between the total score of the combined items with each of the sub-scale scores (social and emotional) and between each of the sub-scale scores $(r=0.92-0.98, \rho<0.01)$. Conclusions: The preliminary finding suggests the translated version of HHIA has the potential to be a reliable and valid tool to evaluate the hearing handicaps among hearing-impaired patients in Malaysia. A large sample of subjects is needed for further evaluation to support this notion.

KEYWORDS: hearing handicap inventory for adult, HHIA, outcome measurement, audiology 